DFTUZ/99/01

\title{
Correlation between UV and IR cutoffs in quantum field theory and large extra dimensions
}

\author{
J.L. Cortés* \\ Departamento de Física Teórica, Universidad de Zaragoza, 50009 Zaragoza, Spain
}

(January 22, 1999)

\begin{abstract}
A recently conjectured relashionship between UV and IR cutoffs in an effective field theory without quantum gravity is generalized in the presence of large extra dimensions. Estimates for the corrections to the usual calculation of observables within quantum field theory are used to put very stringent limits, in some cases, on the characteristic scale of the additional compactified dimensions. Implications for the cosmological constant problem are also discussed.
\end{abstract}

04.50.+h, 11.10.Kk

*E-mail: cortes@leo.unizar.es 
Introduction. The main subject of this note is to try to discuss the implications for low energy physics of the breakdown of effective field theory, with an UV cutoff $\Lambda$, to describe a system in a finite volume with a length $L$ when this length becomes smaller than a critical value which depends on the UV cutoff. One way to derive a limitation of this type on conventional quantum field theory is based on the postulate [1] that the maximum entropy in a box of volume $L^{3}$ is proportional to the area of the box. A stronger constraint on the validity of quantum field theory is obtained [2] if one restricts the IR cutoff $L$ in order to exclude states containing a black hole. Assuming a simple power dependence on the cutoffs for the corrections to a conventional calculation in an infinite box without a UV cutoff one finds [2] that the uncertainty in the quantum field theory calculation is far larger than what one naively would ascribe to gravitational effects.

The second ingredient of the present discussion is the very interesting recent proposal of large compactified extra dimensions [3] which allow to understand the observed weakness of gravity in a theory with only one fundamental short distance scale, the weak scale.

When one considers simultaneously the possibility to have large extra dimensions and also the limitations of quantum field theory due to the impossibility to describe states containing a black hole then it becomes important to try to estimate the errors in any conventional quantum field theory calculation which is the aim of this short note.

Deviations from QFT calculations. Let us assume, to start with, that the length $L$ of the finite box, which acts as an infrared cutoff, is smaller than the compactification radius $R$ so we can consider a box of volume $L^{3+n}$ in $3+n$ space dimensions. A restriction on the infrared cutoff can be obtained [2] by requiring to any state to have a Schwarzschild radius $L_{0}$ smaller than its size $L$. If one denotes by $M$ the mass scale associated to the gravitational constant in $4+n$ dimensions $\left(G=1 / M^{n+2}\right)$ and uses the bound $\rho \leq \Lambda^{4}$ for the energy density in the presence of an UV cutoff $\Lambda$ then one has that the largest possible value for $L_{0}$ is related to the UV cutoff through $L_{0}^{1+n} \sim M^{-(2+n)} L^{3+n} \Lambda^{4+n}$. Then the condition $L_{0}<L$ leads to a restriction combining the IR and UV cutoffs

$$
L^{2} \Lambda^{4+n}<M^{2+n}
$$

which is the generalization of the constraint $L \Lambda^{2}<M_{P}$ obtained [2] in $3+1$ dimensions.

In order to estimate the corrections to a conventional quantum field theory calculation done in an infinite box without a UV cutoff one assumes [2] that they can be given as an expansion in powers of $1 / \Lambda$ and $1 / L$. One can consider two general classes of observables, "chirally" protected $(\hat{O})$ involving corrections with even powers of the cutoffs exclusively and the remaining ones $(O)$ which have all power corrections. The result for an observable $\hat{O}$ including one loop radiative corrections will be

$$
\hat{O} \approx \hat{O}_{Q F T}[1+\delta \hat{O}]
$$

where $\hat{O}_{Q F T}$ is the calculation in QFT including one loop radiative corrections and the correction $\delta \hat{O}$ can be estimated to be

$$
\delta \hat{O} \sim \frac{\alpha}{\pi}\left[\frac{E^{2}}{\Lambda^{2}}+\frac{1}{L^{2} E^{2}}\right] \geq \frac{\alpha}{\pi}\left[\frac{E^{2}}{\Lambda^{2}}+\frac{\Lambda^{4+n}}{E^{2} M^{2+n}}\right]
$$

where $\alpha$ is the coupling of the perturbative expansion and $E$ is the characteristic energy of the observable $\hat{O}$. 
From this estimate for the correction to the field theoretical calculation it is possible to determine the choice for the UV cutoff which minimizes the discrepancy in the calculation of $\hat{O}$ in QFT. The best choice for $\Lambda$ corresponds to $\Lambda^{6+n} \sim E^{4} M^{2+n}$ and the minimal uncertainty in the calculation is

$$
\delta_{\min } \hat{O} \sim \frac{\alpha}{\pi}\left(\frac{E}{M}\right)^{\frac{4+2 n}{6+n}}
$$

In the case of two extra dimensions one has $\delta_{\min } \hat{O} \sim(\alpha / \pi)(E / M)$ which is larger than the corrections that one would expect in an effective field theory calculation valid up to the scale $M$ which signals the onset of gravitational effects, $\delta \hat{O} \sim(\alpha / \pi)(E / M)^{2}$.

If one considers an observable with no "chiral" protection the discussion can be repeated and the final answer is

$$
\delta_{\min } O \sim \frac{\alpha}{\pi}\left(\frac{E}{M}\right)^{\frac{2+n}{6+n}}
$$

which for the case of two extra dimensions gives a correction proportional to $\sqrt{E / M}$ to the QFT calculation.

From the success of the QFT one can derive a lower bound on the scale $M$ of the gravitational coupling in $n+4$ dimensions. The bound will be higher as the deviations of the experimental determination from the QFT calculation is smaller and for a given accuracy one will get more stringent bounds as the energy increases. As an explicit example of a chirallyprotected observable, which is in fact the most important quantitatively, one can consider the anomalous magnetic moment of the electron, $g-2$, which is known to an accuracy of $10^{-11}$. In this case one has a characteristic energy scale $E=m_{e}$ and demanding $\delta_{\min }(g-2)$ to be smaller than the experimental error leads, in the case of two extra dimensions, to the bound $M>100 \mathrm{TeV}$. This bound is more stringent that the bound obtained previously by considering contributions of Kaluza-Klein (KK) modes. The very weak coupling of KKgravitons is compensated by their very large multiplicity [4] and one finds [5, 何] a cross section for emission of KK-gravitons of the order of $E^{2} / M^{4}$ where $E$ is the center-of-mass energy and a corresponding lower bound $M>30 \mathrm{TeV}$.

If one considers the anomalous magnetic moment of the muon one has $E=m_{\mu}$ and then larger corrections than in the case of the electron but also the experimental error is larger $\left(\sim 10^{-8}\right)$ so that the corresponding bound for $M$ is lower. The same happens for other observables at higher energies which are not determined experimentally with enough precission to give a bound comparable with the one obtained from $(g-2)_{e}$.

If one considers a higher number of extra dimensions then the exponent of $E / M$ in the deviations from QFT is bigger and then one finds smaller values for the lower bound on the graviational mass scale $M$. In the particular case of six extra dimensions one finds $M \gtrsim 1 \mathrm{TeV}$ which is closer to the Fermi scale.

Consistency of the determination of $\delta_{\min } \hat{O}$. In the discussion of deviations from the QFT conventional calculation we have assumed that the compactification radius $R$ in the extra dimensions is smaller than the lenght $L$ of the box. The relation [3, 4] between the $4+n$-dimensional gravitational coupling and the effective four-dimensional Newton constant, $R^{n} M^{2+n}=M_{P}^{2}$ where $M_{P}$ is the Planck mass scale, can be used to determine the radius $R$ in terms of the scale $M$, 


$$
R^{2}=\frac{1}{M^{2}}\left(\frac{M_{P}}{M}\right)^{4 / n}
$$

The result(4) for the minimal deviation from the QFT calculation is obtained with an UV cutoff $\Lambda$ such that $\Lambda^{6+n} \sim E^{4} M^{2+n}$ and an IR cutoff $L$ such that $L^{2} \sim M^{2+n} / \Lambda^{4+n}$. Then one has

$$
L^{2} \sim \frac{1}{M^{2}}\left(\frac{M}{E}\right)^{\frac{16+4 n}{6+n}}
$$

The condition $L<R$ gives a restriction on the energy scale $E$,

$$
E>M\left(\frac{M}{M_{P}}\right)^{\frac{6+n}{n(4+n)}}
$$

which in the case $n=2$ can be rewritten as

$$
E \gtrsim \frac{m_{e}}{10}\left(\frac{M}{100 T e V}\right)^{5 / 3}
$$

Then in the case of two large extra dimensions with $M \sim 100 \mathrm{TeV}$ we have that the estimate (4) for the deviations from QFT is valid for all proccesses with an energy scale $E \gtrsim 100 \mathrm{KeV}$. This includes all the applications of relativistic quantum field theory.

If one considers more than two extra dimensions then the range of energies (8) where the estimate (4) for the deviations from QFT is valid is reduced. In the particular case of six extra dimensions one has

$$
E>M\left(\frac{M}{M_{P}}\right)^{1 / 5} \sim 1 G e V\left(\frac{M}{1 T e V}\right)^{6 / 5},
$$

which in this case excludes, for $M$ close to the Fermi scale, all the applications of QFT at energies $E \lesssim 1 G e V$. This includes the evaluation of $(g-2)$ for the electron where $E=m_{e}$. At this very low energy the IR cutoff which minimizes the deviation from the QFT evaluation is such that $L \gg R$ and then one can neglect the extra dimensions in the estimate of the deviations from QFT. In this case one has to replace $n$ by $n_{e f f}=0$ and $M$ by $M_{e f f}=M_{P}$ in (4) and one has [2]

$$
\delta_{\min }\left[(g-2)_{e}\right] \sim \frac{\alpha}{\pi}\left(\frac{m_{e}}{M_{P}}\right)^{2 / 3} \sim \frac{\alpha}{\pi} \times 10^{-15},
$$

which is very small compared with the experimental error in the determination of $(g-2)_{e}$. The stronger constraint on the scale $M$ will come, in this case, from high energy observables $E \gtrsim 1 \mathrm{GeV}$, where one expects deviations from QFT of the order of

$$
\delta_{m i n} \hat{O} \sim \frac{\alpha}{\pi}\left(\frac{E}{M}\right)^{4 / 3} .
$$


Cosmological constant. We end up with a comment on the result of a QFT evaluation of the vacuum energy density $\rho_{0}$ along the same lines. The result in perturbation theory will be $\rho_{0} \sim \Lambda^{4}$ and in order to reproduce the value suggested by the supernovae data an UV cutoff $\Lambda \sim 2.5 \times 10^{-3} \mathrm{eV}$ is required 四. If one uses the relation $R=M_{P} / M^{2}$ between the compactification radius $R$ and the scale $M$ and the relation between the cutoffs leading to $\delta_{\min } \hat{O}$ in the case of two extra dimensions $\left(L \Lambda^{3} \sim M^{2}\right)$ then one finds for the IR cutoff

$$
L \sim 10^{36}\left(\frac{M}{100 \mathrm{TeV}}\right)^{4} R
$$

which is much bigger than $R$. In this case one would expect that the extra dimensions can be neglected and one should repeat all the considerations based on a correlation between IR and UV cutoffs but now in four effective space-time dimensions. In this case the UV cutoff which minimizes the deviations from a QFT calculation is $\Lambda \sim\left(E^{2} M_{P}\right)^{1 / 3}$; then in order to reproduce the value of the UV cutoff, $\Lambda \sim 2.5 \times 10^{-3} \mathrm{eV}$, and then the smallness of the cosmological constant, one has to find a characteristic energy scale in the evaluation of the vacuum energy density $E_{0} \sim 10^{-18} \mathrm{eV}$ (!). It is not clear what can be the origin of such a small energy scale. Alternatively one can estimate the corresponding value of the effective IR cutoff through $L_{e f f} \Lambda^{2} \sim M_{P}$ which gives $L_{e f f} \sim 10^{28} \mathrm{~cm}$, a value comparable to the present horizon size. In order to predict what the cosmological constant should be one would have to go beyond QFT to find the origin of these scales.

I am grateful to M. Asorey, F. Falceto and A. Segui for discussions and J.L. Alonso for reading the manuscript. This work was supported by CICYT (Spain) project AEN-97-1680. 


\section{REFERENCES}

[1] J.D. Bekenstein, Phys. Rev. D23, 287 (1981).

[2] A.G. Cohen, D.B. Kaplan and A.E. Nelson, hep-th/9803132.

[3] I. Antoniadis, Phys. Lett. B246, 377 (1990); N. Arkani-Hamed, S. Dimopoulos and G. Dvali, Phys. Lett. B429, 263 (1998); K.R. Dienes, E. Dudas and T. Gherghetta, Phys. Lett. B436, 55 (1998).

[4] S. Nussinov and R. Shrock, hep-ph/9811323.

[5] N. Arkani-Hamed, S. Dimopoulos and G. Dvali, hep-ph/9807344. 